\title{
Características seminais pós-descongelamento em garanhões da raça crioula
}

Post-thaw seminal characteristics of Criollo breed stallions

\author{
Verônica La Cruz Bueno ${ }^{1,2, *}$, Leonardo Glaeser Paul ${ }^{1}$, Henrique Boll de Araujo Bastos ${ }^{1}$, Gustavo \\ Rupp Larentis ${ }^{1}$, Rodrigo Costa Mattos ${ }^{1,2}$, Sandra Fiala Rechsteiner ${ }^{1,2}$. \\ ${ }^{1}$ REPROLAB - Faculdade de Veterinária, UFRGS, Porto Alegre-RS, Brasil; \\ ${ }^{2}$ HISTOREP - Instituto de Biologia, Universidade Federal de Pelotas, UFPEL, Pelotas-RS, Brasil
}

\begin{abstract}
Resumo
Este trabalho teve como objetivo avaliar as características seminais de garanhões da raça Crioula após o processo de criopreservação utilizando o BotuCrio ${ }^{\circledR}$. Foram utilizados 24 garanhões alojados nas proximidades de Porto Alegre, RS, Brasil. As análises do sêmen foram realizadas pré e pósdescongelamento através do sistema Computer Assisted Sperm Analysis AndroVision ${ }^{\circledR}$. Somente foram utilizadas amostras com motilidade total $\geq 60 \%$ e vigor $\geq 3$, estas foram envasadas em palhetas de 0,5 $\mathrm{ml}$, dispostas horizontalmente a $5^{\circ} \mathrm{C}$ durante 20 minutos. Após colocadas a $6 \mathrm{~cm}$ acima do nível de nitrogênio líquido durante 20 minutos. Sendo finalmente imersas em nitrogênio líquido, após descongeladas em banho-maria a $37^{\circ} \mathrm{C}$ durante 30 segundos. Os valores médios e desvios padrões das variáveis pós-descongelamento foram: motilidade total $(52,85 \% \pm 7,27)$; motilidade progressiva $(31,15$ $\% \pm 10,21)$; motilidade rápida $(4,78 \% \pm 5,37)$; motilidade local $(22,44 \% \pm 8,68)$; velocidade curvilinear $(97,67 \mu \mathrm{m} / \mathrm{s} \pm 35,25)$; velocidade em linha reta $(35,33 \mu \mathrm{m} / \mathrm{s} \pm 15,29)$; velocidade da trajetória média $(44,99 \mu \mathrm{m} / \mathrm{s} \pm 17,98)$; linearidade $(0,3 \% \pm 0,15)$; BCF $(2,5 \mathrm{~Hz} \pm 1,7)$; $\mathrm{ALH}(0,35 \mu \mathrm{m} \pm 0,3)$; integridade funcional da membrana $(42,73 \% \pm 7,06)$ e integridade estrutural da membrana $(48,06 \% \pm 8,99)$. Concluímos que o protocolo utilizando diluente BotuCrio ${ }^{\circledR}$ é viável para criopreservação do sêmen de cavalos da raça Crioula, pois garante motilidade acima de $30 \%$ pós-descongelamento que é recomendado pelo Colégio Brasileiro de Reprodução Animal.
\end{abstract}

Palavras chaves: espermatozoides, diluente, BotuCrio $^{\circledR}$, equino.

\begin{abstract}
The aim of this study was to evaluate seminal characteristics of Criollo breed stallions, after cryopreservation process using the BotuCrio ${ }^{\circledR}$. One ejaculate from twenty-four stallions located near to Porto Alegre, RS, Brazil were used. Semen analysis were performed pre and post-freezing through the Computer Assisted Sperm Analysis AndroVision ${ }^{\circledR}$ system. Only samples with total motility $\geq 60 \%$ and vigor $\geq 3$ were used. The samples were placed in $0.5 \mathrm{ml}$ straws, arranged horizontally at $5^{\circ} \mathrm{C}$ for 20 minutes. Then, $6 \mathrm{~cm}$ above the level of liquid nitrogen in vapor for 20 minutes. Finally, being immersed in liquid nitrogen, the samples thawed in a water bath at $37^{\circ} \mathrm{C}$ for 30 seconds. Mean values and standard deviations of post-freeze variables were: total motility (52.85\% \pm 7.27); progressive motility (31.15\% \pm 10.21); rapid motility (4.78\% 5.37); local motility (22.44\% $\%$ 8.68); Curvilinear Velocity (97.67 $\mu \mathrm{m} / \mathrm{s}$ \pm 35.25); Straight Line Velocity (35.33 $\mu \mathrm{m} / \mathrm{s} \pm 15.29) ;$ Average Path Velocity (44.99 $\mu \mathrm{m} / \mathrm{s} \pm 17.98)$;

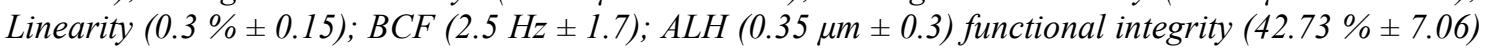
and physical integrity (48.06\% \pm 8.99$)$. We conclude that the protocol used for semen cryopreservation is feasible for use in the Criollo breed, because it has motility above $30 \%$ post-freezing semen parameters, wich is the recommended by the Brazilian College of Animal Reproduction.
\end{abstract}

Keywords: sperm, extender, BotuCrio ${ }^{\circledR}$, equine.

\section{Introdução}

Atualmente mais de 400 mil animais possuem registro na Associação Brasileira de Criadores de Cavalos Crioulos (ABCCC, 2019). O crescimento do uso da inseminação artificial (IA) com sêmen congelado é decorrente da liberação do uso por diversas associações de criadores, sendo que a Associação Brasileira de Criadores da Raça Crioula permitiu a utilização desta biotécnica em 2011 (ABCCC, 2019; Alvarenga e Leão, 2002).

Correspondência: veronicalacruzbueno@hotmail.com Recebido: 20 de agosto de 2019

Aceito: 15 de abril de 2020 
A utilização de sêmen congelado possibilita primariamente a manutenção e utilização do sêmen por tempo indeterminado, maximizando o potencial genético de garanhões zootecnicamente superiores e reduzindo consideravelmente os custos relacionados ao deslocamento das éguas. Também contribui para redução da transmissão de doenças contagiosas; possibilita a continuidade de estações reprodutivas em caso de lesões ou participação do garanhão em competições; minimiza o uso de garanhões geneticamente inferiores (Amann e Pickett, 1987; Miller, 2008). Embora esta biotécnica seja acompanhada por diversos benefícios, muitos garanhões não obtêm taxas satisfatórias de prenhez (Amann e Pickett, 1987), sendo necessários, dois a três ciclos e grande número de doses para obter uma prenhez (Alvarenga e Leão, 2002). Esta desvantagem, em muitos casos, pode ser superada pela adoção de diferentes técnicas, tornando-se insignificante quando comparada às inúmeras vantagens do sêmen criopreservado (Miller, 2008).

A qualidade do sêmen depende da integridade e função de todas as estruturas que compõem a célula espermática, que nem sempre correspondem à capacidade fecundante do espermatozoide (Arruda et al., 2011). O sucesso da criopreservação está associado diretamente com as diferenças inerentes ao espermatozoide que permitem sua sobrevivência durante o processo de congelamento (Loomis e Graham, 2008). Estas diferenças não são notadas apenas entre diferentes espécies, mas também entre machos de uma mesma espécie (Darin-Bennett e White, 1977), em especial na espécie equina, onde a variação individual é uma característica marcante (Aurich, 2008).

A fertilidade ou o potencial de fertilização são características, na melhor das hipóteses, secundárias na escolha de um garanhão para reprodutor (Colenbrander et al., 2003). Os garanhões, diferentemente de outras espécies de interesse zootécnico, são selecionados pelo desempenho esportivo e pela sua genealogia (Varner et al., 2008), o aspecto reprodutivo na maioria das vezes não é levado em consideração (Sieme e Distl, 2012).

$\mathrm{Na}$ maioria das espécies, incluindo a espécie equina, o glicerol é o crioprotetor penetrante (intracelular) utilizado como eletivo em espermatozoides (Amann e Pickett, 1987; Oldenhof et al., 2017). O efeito de tal crioprotetor na membrana plasmática ocorre por meio de uma ligação direta aos fosfolipídios, reduzindo sua fluidez e interferindo na permeabilidade celular (Parks e Graham, 1992). No entanto, a baixa fertilidade do sêmen congelado de alguns garanhões é relacionada à presença do glicerol nos meios de congelamento (Squires et al., 2004).

Alvarenga et al., (2005), mencionam que o glicerol pode reduzir as taxas de fertilidade do sêmen congelado mesmo quando a motilidade e a viabilidade de espermatozoides são preservadas. Neste contexto, crioprotetores menos tóxicos que o glicerol estão sendo estudados como alternativas durante o congelamento de sêmen. Em estudos realizados com sêmen equino foram relatadas evidências sobre a proteção das amidas no processo de criopreservação, capacidade ainda mais notável quando o sêmen processado provém de garanhões que congelam mal quando utilizado glicerol (Medeiros et al., 2002; Squires et al., 2004).

As amidas podem induzir um menor dano osmótico ao penetrar na membrana plasmática mais rapidamente, devido ao menor peso molecular quando comparadas ao glicerol (Alvarenga et al., 2005; Squires et al., 2004). A crioproteção da célula espermática através do uso de amidas, como a dimetilformamida (Alvarenga et al., 2005; Medeiros et al., 2002; Oldenhof et al., 2017; Squires et al., 2004), inclusive em garanhões da raça crioula (Oliveira et al., 2013), a metilformamida (Alvarenga et al., 2005; Medeiros et al., 2002; Squires et al., 2004) e a dimetilacetamida (Alvarenga et al., 2005), foi demonstrada com eficácia em relação ao glicerol. Além da obtenção de boas taxas de criopreservação, maior fertilidade foi observada utilizando um crioprotetor do grupo amida, comparado ao glicerol com dimetilformamida, em alguns estudos na espécie equina (Medeiros et al., 2002; Moffet et al., 2003).

A fim de que a criopreservação do sêmen equino seja bem-sucedida, é essencial a interação do crioprotetor, do diluidor, da curva de resfriamento e do aquecimento no processo de criopreservação. Se for realizada de maneira correta, a criopreservação minimiza os efeitos deletérios, como o choque térmico, a formação de cristais de gelo e a desidratação celular (Oliveira et al., 2013). Este trabalho teve como objetivo avaliar as características seminais de garanhões da raça Crioula após o processo de criopreservação utilizando o BotuCrio ${ }^{\circledR}$.

\section{Material e Métodos}

Foram utilizados 24 garanhões da raça Crioula, com idade entre 4 e 18 anos, com peso entre 450 e $500 \mathrm{~kg}$, alimentados com concentrado e feno de alfafa diariamente, água e sal ad libitum. Os garanhões estavam em regime de coleta, dos quais foi coletado um ejaculado. Todos os animais localizavam-se nas 
proximidades de Porto Alegre $\left(30^{\circ} \mathrm{S}, 51^{\circ} \mathrm{W}\right)$, RS, Brasil.

Todos os procedimentos experimentais estão de acordo com as diretrizes do Conselho Nacional de Controle de Experimentação Animal (CONCEA) e aprovados pelo Comitê de Ética e Experimentação Animal (CEEA) da Universidade Federal de Pelotas com número 2753-2018.

Para a coleta do sêmen dos animais, utilizou-se vagina artificial modelo Hannover com lubrificação e pré-aquecimento $\left(42-45^{\circ} \mathrm{C}\right)$. Após a coleta, o ejaculado foi filtrado e encaminhado para o Laboratório de Reprodução Animal (REPROLAB) da Faculdade de Veterinária da Universidade Federal do Rio Grande do Sul, com diluente comercial BotuSêmen ${ }^{\circledR}$ (Botupharma, Botucatu, SP, Brasil), com limite máximo de duas horas de transporte.

A concentração espermática foi avaliada por meio de câmara de Neubauer com sêmen fresco antes da centrifugação (Brito et al., 2007). As avaliações de motilidade total (\%) (MT), motilidade progressiva $(\%)(\mathrm{MP})$, motilidade progressiva rápida $(\%)(\mathrm{MR})$, motilidade progressiva lenta $(\%)(\mathrm{ML})$, motilidade local (\%) (MC), imóveis (\%) (IM); velocidade da trajetória média (VAP, $\mu \mathrm{m} / \mathrm{s})$, velocidade em linha reta (VSL, $\mu \mathrm{m} / \mathrm{s}$ ), velocidade curvilinear (VCL, $\mu \mathrm{m} / \mathrm{s}$ ), linearidade (\%) (LIN), frequência de batimento do flagelo (BCF, Hz), amplitude do deslocamento lateral da cabeça (ALH, $\mu \mathrm{m} / \mathrm{s}$ ), foram realizadas através do sistema CASA (Computer Assisted Sperm Analysis, AndroVision ${ }^{\circledR}$, Minitube, Tiefenbach, Alemanha). Os padrões utilizados para o ajuste do equipamento foram: 30 imagens/segundo com $60 \mathrm{~Hz}$; tamanho de partícula capturado entre 4 e $75 \mu \mathrm{m} / \mathrm{m}^{2}$; espermatozoides considerados imóveis $<10 \mu \mathrm{m} / \mathrm{s}$, lentos $<45 \mu \mathrm{m} / \mathrm{s}$, médios de 45 a $80 \mu \mathrm{m} / \mathrm{s}$ e rápidos acima de $80 \mu \mathrm{m} / \mathrm{s}$.

A integridade funcional da membrana plasmática foi avaliada por meio do teste hiposmótico (HOST) realizado conforme Lagares et al. (1998). A análise da integridade estrutural da membrana plasmática foi realizada por meio de fluorescência conforme Garner et al. (1986).

As amostras, após confirmação de motilidade total $\geq 60 \%$ e vigor $\geq 3$, foram submetidas à centrifugação de 600 x $g$ por 10 minutos e o sobrenadante foi removido. O pellet formado foi ressuspendido com diluente comercial BotuCrio ${ }^{\circledR}$ (Botupharma, Botucatu, SP, Brasil) próprio para congelamento, até a concentração de 200 milhões de espermatozoides por mililitro ( $\mathrm{ml})$.

As amostras foram envasadas em palhetas de $0,5 \mathrm{ml}$ e lacradas com álcool polivinílico. A primeira etapa do congelamento foi realizada em refrigerador comercial, as palhetas foram dispostas horizontalmente a $5^{\circ} \mathrm{C}$ durante 20 minutos. Logo após, as palhetas foram acondicionadas horizontalmente em estante específica a $6 \mathrm{~cm}$ acima do nível de nitrogênio líquido em caixa de isopor para congelamento no vapor de nitrogênio durante 20 minutos. Sendo finalmente imersas em nitrogênio líquido (Papa et al., 2008). Para fins de avaliação as amostras de sêmen foram descongeladas em banho-maria a $37^{\circ} \mathrm{C}$ durante 30 segundos. O sêmen descongelado foi submetido aos mesmos métodos de avaliação do sêmen fresco. As amostras foram descongeladas 60 dias após a criopreservação.

Foi realizada a média e desvio padrão de todas as variáveis analisadas do sêmen fresco e pósdescongelamento. O teste de Shapiro-Wilk foi realizado para verificação da normalidade dos dados. Foi realizada analise de variância para dados não paramétricos Kruskal-Wallis e para dados paramétricos foi realizado análise de variância ANOVA seguida do teste T de Tukey.

\section{Resultados}

Os valores médios e desvios padrão das variáveis pré-congelamento (sêmen fresco, transportado com limite máximo de 2 horas) obtidos foram: concentração espermática de $250 \times 10^{6}$ milhões $/ \mathrm{ml} \pm$ 135,34; MT de 75,93\% $\%$ 10,27; MP de 53,71\% \pm 18,38; MR de 13,78 \% $\pm 10,38$; ML de 32,43\% \pm 13,37; MC de 22,43 \% $\pm 10,17$; VCL de 116,88 $\mu \mathrm{m} / \mathrm{s} \pm 42,34$; VSL de 44,79 $\mu \mathrm{m} / \mathrm{s} \pm 17,72$; VAP de $56,40 \mu \mathrm{m} / \mathrm{s} \pm 21,08$; LIN de 0,4 $\mu \mathrm{m} / \mathrm{s} \pm 0,09$; BCF de 3,9 Hz $\pm 1,6$; ALH de 0,7 $\mu \mathrm{m} \pm 0,6$; HOST de $63,09 \% \pm 14,02$ e integridade estrutural de membrana de $63,04 \% \pm 10,45$.

Os valores médios e desvios padrão das características espermáticas pós-descongelamento foram: MT de 52,85 \% 7,27; MP de 31,15\% $\pm 10,21$; MR de 4,78 \% \pm 5,37; ML de 24,89 \% $\pm 8,17$; $\mathrm{MC}$ de 22,44 \% 18,$68 ; \mathrm{VCL}$ de $97,67 \mu \mathrm{m} / \mathrm{s} \pm 35,25 ; \mathrm{VSL}$ de $35,33 \mu \mathrm{m} / \mathrm{s} \pm 15,29 ; \mathrm{VAP}$ de $44,99 \mu \mathrm{m} / \mathrm{s} \pm$ 17,98; LIN de 0,3\% 0,15 ; BCF de $2,5 \mathrm{~Hz} \pm 1,7$; ALH de 0,35 $\mu \mathrm{m} \pm 0,3$; HOST de 42,73 \% \pm 7,06 e integridade estrutural da membrana de $48,06 \% \pm 8,99$. Na figura 1 estão expostos os resultados da motilidade total, progressiva e integridade de membranas plasmática, as quais foram estatisticamente menores nas amostras criopreservadas. Nas figuras 2, 3 e 4 estão expostos os resultados cinéticos dos espermatozoides avaliados pelo sistema CASA que é possível comparar as amostras de sêmen fresco e depois do processo de criopreservação. 


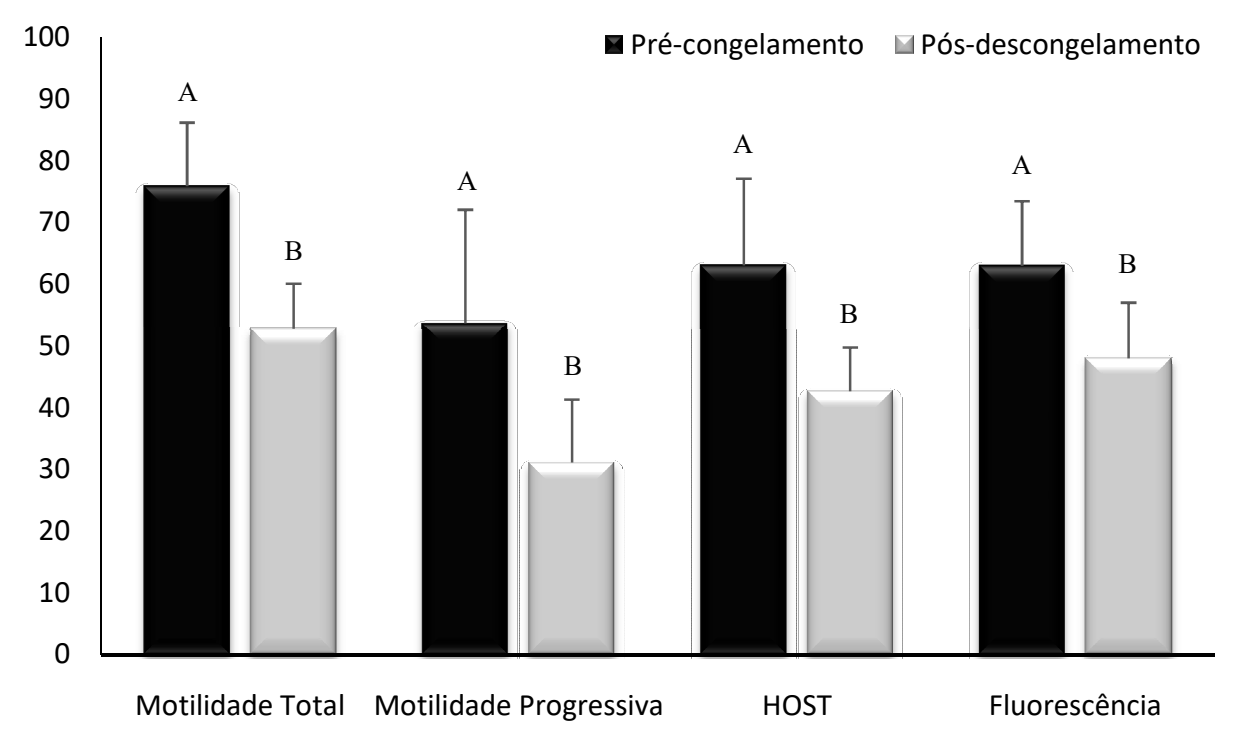

Figura 1. Médias $(\% \pm \mathrm{DP})$ dos resultados da análise de cinética espermática, teste hiposmótico (HOST) e integridade estrutural da membrana plasmática (Fluorescência). As letras A e B indicam diferença estatística entre as diferentes análises pré-congelamento e pós-descongelamento $(\mathrm{P}<0,05)$.

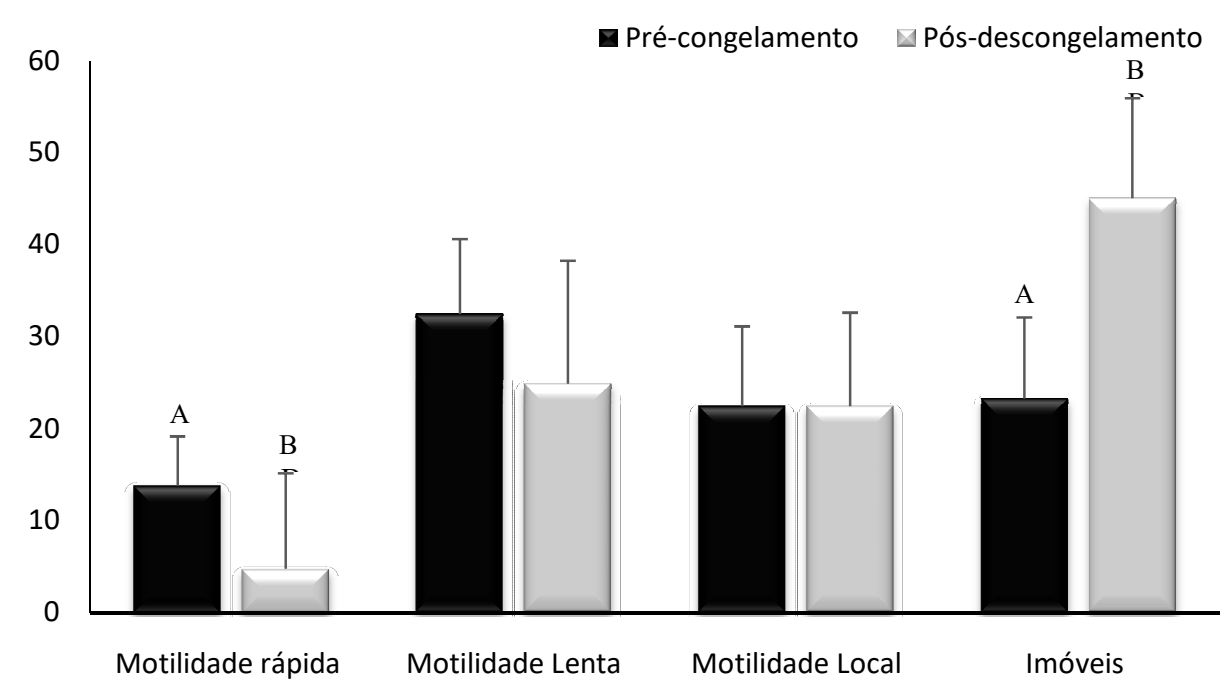

Figura 2. Médias $(\% \pm \mathrm{DP})$ dos resultados da análise de cinética espermática por meio do sistema CASA. As letras A e $\mathrm{B}$ indicam diferença estatística entre as diferentes analises pré-congelamento e pós-descongelamento $(\mathrm{P}<0,05)$. 


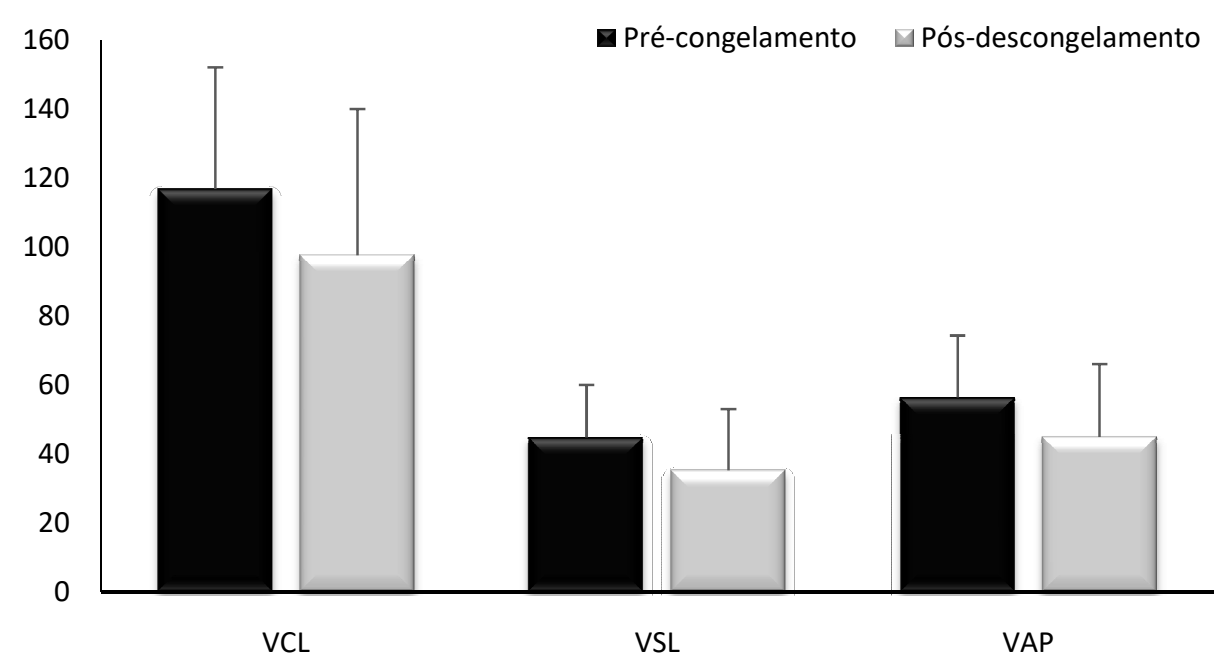

Figura 3. Médias dos resultados da análise de cinética espermática através do sistema CASA. Velocidade curvilínea (VCL), velocidade em linha reta (VSL), velocidade média da trajetória (VAP).

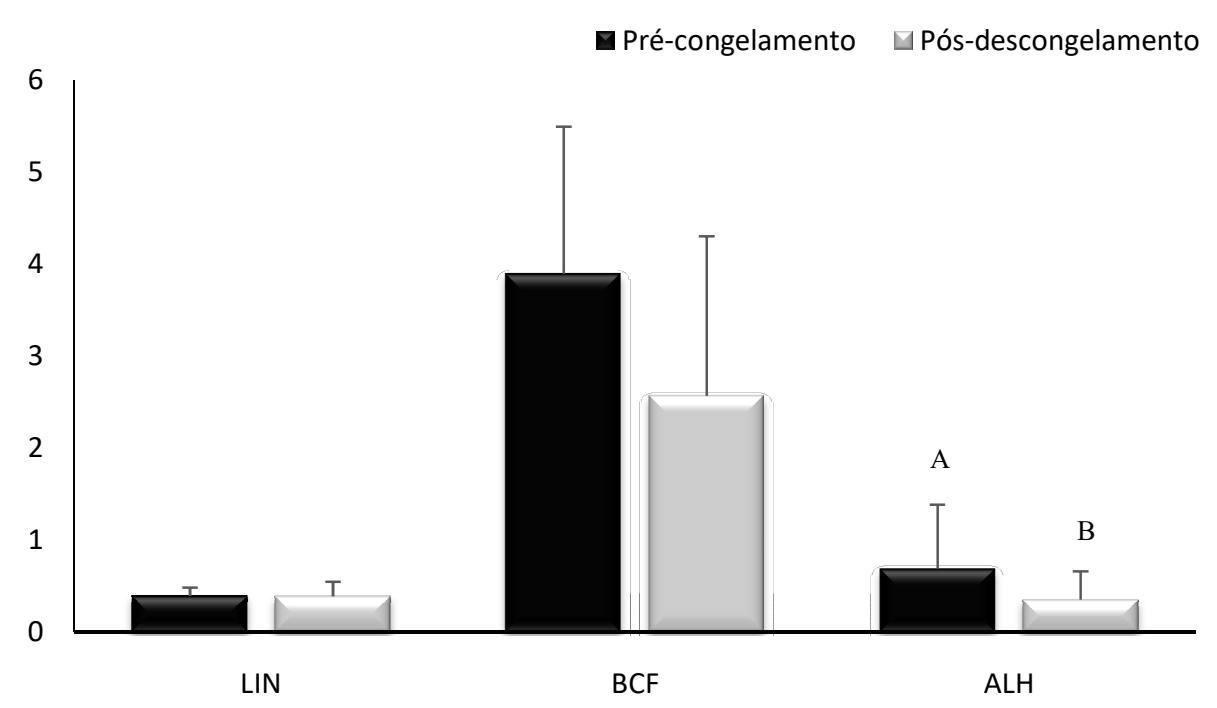

Figura 4. Médias dos resultados da análise de cinética espermática através do sistema CASA. Linearidade (LIN), frequência de batimento do flagelo (BCF), amplitude do deslocamento lateral da cabeça (ALH). As letras A e B indicam diferença estatística entre as diferentes análises pré-congelamento e pós-descongelamento $(\mathrm{P}<0,05)$.

\section{Discussão}

O presente trabalho apresenta dados sobre as características de criopreservação seminal de animais da raça Crioula, sendo que os métodos utilizados foram eficientes para garantir uma boa qualidade seminal pós-descongelamento. Somente foram criopreservados ejaculados que apresentavam motilidade total $\geq 60 \%$ e vigor $\geq 3$ a fresco, como descrito pelo CBRA (2013), possibilitando que o efeito individual do garanhão fosse mitigado. 
Alvarenga et al. (2005), demonstraram que o uso de diluidores com dimetilformamida e metilformamida em garanhões com sêmen de congelabilidade satisfatória pode não resultar em aumento na motilidade dos espermatozóides pós-descongelamento. Os crioprotetores não penetrantes (extracelulares) também podem ser utilizados com intuito de minimizar o estresse osmótico no decorrer do resfriamento. Representados por moléculas de alto peso molecular, como alguns açúcares, lipídios e lipoproteínas da gema de ovo, e proteínas do leite, eles protegem a célula através de mecanismos osmóticos, sem necessidade de penetração (Amann e Pickett, 1987), como o diluente utilizado em nosso trabalho.

A motilidade espermática é comumente apontada como uma das mais importantes características associadas com a habilidade fertilizante do espermatozoide (Cox et al., 2006). Para que a inseminação artificial resulte em prenhez é necessário que uma dose inseminante contenha um número suficiente de espermatozoides viáveis capazes de alcançar o local de fertilização (Morrell e Rodriguez-Martinez, 2009). A MT pós-descongelamento é o parâmetro mais utilizado para selecionar ejaculados após o descongelamento (Vidament et al., 1998). Os resultados encontrados para motilidade total neste estudo estão adequados aos parâmetros pré-estabelecidos (CBRA, 2013).

Os resultados de MT pós-descongelamento foram semelhantes ao encontrado por Candeias et al. (2012) na raça Mangalarga Marchador e Maziero et al. (2019) em garanhões da raça Brasileiro de Hipismo. Os parâmetros iniciais do presente estudo coincidiram com os valores de MT e MP do sêmen fresco (Candeias et al., 2012; Maziero et al., 2019).

Os resultados de VAP, VSL e VCL não apresentaram diferenças significativas entre os grupos, porém foram menores do que o encontrado por Maziero et al. (2019), o crioprotetor utilizado foi o mesmo, porém este resultado divergente pode ter relação com o número de animais, pois foram utilizados 91 ejaculados de 5 garanhões.

Foi encontrada diferença na análise do ALH entre os grupos estudados, o grupo pósdescongelamento apresentou um decréscimo em comparação ao pré-congelamento. Sugerindo que o protocolo utilizado neste estudo foi eficiente, pois os espermatozoides não apresentavam pré-capacitação. As alterações no padrão de movimento espermático podem indicar o início da capacitação espermática precoce, o que não seria desejado (Celeghini et al., 2017). A exposição das células espermáticas à temperatura abaixo das fisiológicas, mesmo antes de ocorrer a congelação, é responsável por mudanças na organização bidimensional dos lipídeos da membrana e também por alterações das propriedades de enzimas encontradas na membrana, diminuindo a longevidade dos espermatozoides após a descongelação, através do adiantamento da capacitação espermática (Holt, 2000). De acordo com Arruda (2000), um maior valor numérico de ALH se traduz em pior qualidade espermática, e desta forma, o deslocamento lateral da cabeça do espermatozoide não é desejado, pois pode interferir na progressão da célula (Celeghini et al., 2007).

Os resultados encontrados no teste de integridade estrutural da membrana plasmática pósdescongelamento foram semelhantes aos encontrados por Maziero et al. (2019) e Hernández-Avilés et al. (2018) em garanhões da raça Passo Fino Colombianos. A estrutura da membrana plasmática é muito importante sob a perspectiva do choque térmico (Desai et al., 2010). Além das lesões sofridas durante a criopreservação, também ocorrem danos durante o processo de descongelamento da célula, uma vez que a membrana é submetida a rearranjos estruturais envolvendo lipídios e proteínas, e a passagem rápida de água para o interior da célula pode causar o seu rompimento (Holt, 2000). Sua integridade é fundamental para que a célula espermática esteja viável no momento da fecundação evitando o choque térmico e mantendo a fluidez da membrana plasmática (Bueno et al., 2019).

$\mathrm{Na}$ literatura, a escolha do crioprotetor parece ter sido uma questão de tentativa e erro devido a não existir uma explicação completa e satisfatória para a ação dos crioprotetores (Holt, 2000). Tais crioprotetores atuam aumentando a osmolaridade do meio extracelular, ou seja, tornam o ambiente hipertônico promovendo a desidratação pela saída de água do interior da célula espermática de modo a reduzir a formação de cristais de gelo intracelular no processo (Squires et al., 2004).

Para que ocorra o desenvolvimento da IA e seja possível a comparação, a interpretação e a reprodução de resultados, muitos autores têm mencionado a necessidade da padronização de procedimentos para manipulação do sêmen.

\section{Conclusão}

Desde que a IA foi liberada pela Associação Brasileira de Criadores de Cavalos Crioulos sua utilização foi amplamente difundida. Este trabalho apresenta as características seminais pós- 
descongelamento de garanhões da raça Crioula. Os resultados demostram que o protocolo para criopreservação com o diluente comercial BotuCrio ${ }^{\circledR}$ é indicado para esta raça.

\section{Referências}

ABCCC. Associação Brasileira de Criadores de Cavalo Crioulo. Disponível em $<$ http://www.cavalocrioulo.org.br/studbook/cavalo_crioulo $>$. Acesso em jun. de 2019.

Alvarenga MA, Leão KM. Hysteroscopic insemination of mares with low number of frozen thawed spermatozoa selected by percoll gradient. Theriogenology, p.651-653, 2002.

Alvarenga MA, Papa FO, Landim-Alvarenga FC, Medeiros ASL. Amides as cryoprotectants for freezing stallion semen: A review. Anim Reprod Sci, v.89, p.105-113, 2005.

Amann RP, Pickett BW. Principles of cryopreservation and a review of cryopreservation of stallion spermatozoa. J Equine Vet Sci, v.7, n.3, p.145-173, 1987.

Arruda RP, Celeghini ECC, Alonso MA, Carvalho HF, Oliveira LZ, Nascimento J, Silva DF, Affonso FJ, Lemes KM, Jaimes JD. Métodos de avaliação da morfologia e função espermática: momento atual e desafios futuros. Rev Bras Reprod Anim, v.35, p.145-151, 2011.

Aurich C. Recent advances in cooled-semen technology. Anim Reprod Sci, v.107, p.268-75, 2008.

Brito LFC. Evaluation of Stallion Sperm Morphology. Clin Tech Equine Pract, v.6, p.249-264, 2007.

Bueno VLC, Bastos HBA, Centeno LAM Kretzmann Filho NA, Mattos RC, Rechsteiner SF. Expressão do TNF- $\alpha$ no espermatozoide equino e sua relação com a qualidade seminal póscongelamento. Ver Bras Reprod Anim, v.43, n.2, p.599, 2019.

Candeias ML, Alvarenga MA, Carmo MT, Ferreira HF, Maior MRS, Torres RAF, Rodrigues ALR, Brandão FZ. Semen cryopreservation protocols of Mangalarga Marchador stallions. Rev Bras Zootec, v.41, n.9, p.1989-1995, 2012.

Celeghini ECC, Arruda RP, Andrade AFC, Nascimento J, Raphael CF. Practical techniques for bovine sperm simultaneous fluorimetric assessment of plasma, acrosomal and mitochondrial membranes. Reprod Domest Anim, v.42, p.479-488, 2007.

Celeghini ECC, Arruda RP, Florez-Rodriguez SA, Santos FB, Alves MBR, Oliveira BMM. Impacto da qualidade do sêmen sobre a fertilidade a campo em bovinos. Rev Bras Reprod Anim, v.41, n.1, p.4045, 2017.

Colenbrander B, Gadella BM, Stout TAE. The predictive value of semen analysis in the evaluation of stallion fertility. Reprod Domest Anim, v.38, p.305-311, 2003.

Cox JF, Alfaro V, Montenegro V, Rodriguez-Martinez H. Computer-assisted analysis of sperm motion in goats and its relationship with sperm migration in cervical mucus. Theriogenology, v.66, p. 860-867, 2006.

Darin-Bennett A, White IG. Influence of the cholesterol content of mammalian spermatozoa on susceptibility to cold-shock. Cryobiology, v.14, n.4, p.466-470, 1977.

Desai NR, Mahfouz R, Sharma R, Gupta S, Agarwal A. Reactive oxygen species levels are independent of sperm concentration, motility, and abstinence in a normal, healthy, proven fertile man: a longitudinal study. Fertil Steril, v.94, p.1541-1543, 2010.

Garner DL, Pinkel D, Johnson LA, Pace MM. Assessment of spermatozoal function using dual fluorescent staining and flow cytometric analyses. Biol Reprod, v.34, n.1, p.127-138, 1986.

Hernández-Avilés C, Gómez-Romero M, Buitrago-Horta R, Lozano-Márquez H, Jiménez-Escobar C e Zambrano-Varón J. Evaluation of post-thaw sperm function and integrity parameters under different freezing regimens in Colombian Paso Fino stallions. J Equine Vet Sci, v.67, p.7-14, 2018.

Holt WV. Basic aspects of frozen storage of semen. Anim Reprod Sci, v.62, p.3-22, 2000.

Lagares MA. Preservação do sêmen fresco equino: Avaliação da integridade da membrana espermática sob condições hiposmóticas. Arq Fac Vet UFRGS, v.26, n.1, p.29-42, 1998.

Loomis PR, Graham JK. Commercial semen freezing: individual male variation in cryosurvival and the response of stallion sperm to customized freezing protocols. Anim Reprod Sci, v.105, p.119-128, 2008.

Maziero RRD, Guaitolini CRF, Monteiro GA, Martin I, Silva JPM, Crespilho AM, PaPa FO. Effect of using two cryopreservation methods on viability and fertility of frozen stallion sperm. J Equine Vet Sci, v.72, p. 37-40, 2019.

Medeiros ASL, Gomes GM, Carmo MT, Papa FO, Alvarenga MA. Cryopreservation of stallion sperm using different amides. Theriogenology, v.58, p.273-276, 2002.

Miller CD. Optimizing the use of frozen-thawed equine semen. Theriogenology, v.70, n.3, p.463-468, 2008. 
Moffet PD, Bruemmer JE, Card C, Squires EL. Comparison of dimethyl formamide and glycerol for cryopreservation of equine spermatozoa. Proceedings Society for Theriogenology Annual Conference, 42 (abstract), 2003.

Morrell JM, Rodriguez-Martinez H. Biomimetic techniques for improving sperm quality in animal breeding: a review. TOANDROJ, v.1, p.1-9, 2009.

Oldenhof H, Bigalk J, Hettel C, Barros LO, Sydykov B, Bajcsy AC, Sieme H, Wolkers WF. Stallion sperm cryopreservation using various permeating agents: Interplay between concentration and cooling rate. Biopreserv Biobank, v.15, p.1-10, 2017.

Oliveira RA, Rubin MIB, Silva CAM. Índice de prenhez com sêmen congelado de garanhões da raça crioula usando glicerol ou dimetilformamida como crioprotetores. Ciên Anim Bras, v.14, n.4, p.488-494, 2013.

Papa FO, Melo CM, Fioratti EG, Dell'Aqua-Junior JA, Zahn FS, Alvarenga MA. Freezing of stallion epididymal sperm. Anim Reprod Sci, v.107, p.293-301, 2008.

Parks JE, Graham JK. Effects of cryopreservation procedures on sperm membranes. Theriogenology, v.38, p.209-222, 1992.

Sieme H, Distl O. Genomics and fertility in stallions. J Equine Vet Sci, v.32, p.467-470, 2012.

Squires EL, Keith SL, Graham JK. Evaluation of alternative cryoprotectants for preserving stallion spermatozoa. Theriogenology, v.62, p.1056-1065, 2004.

Varner DD, Brinsco SP, Hartman DL, Carroll BS, Love CC, Blanchard TL, Bliss SB, Eslick MC. Semen processing for the subfertile stallion. J Equine Vet SCI, v.28, p.677-685, 2008.

Vidament M, Cognard E, Yvon JM, Sattler M, Palmer E, Magistrini M. Evaluation of stallion semen before and after freezing. Reprod Domest Anim, v.33, p.271-277, 1998. 